\title{
La Gestión de Tecnologías Emergentes en el Ámbito Universitario
}

\author{
Claudia N. Jiménez-Hernández ${ }^{1}$ \\ Oscar F. Castellanos-Domínguez ${ }^{2}$ \\ Eliana M. Villa-Enciso ${ }^{3}$
}

\section{Resumen}

El carácter emergente de algunas tecnologías hace que tengan un alto grado de incertidumbre, lo que a su vez conlleva particularidades en su manejo respecto a las tecnologías maduras. El objetivo de este artículo es analizar la gestión de tecnologías emergentes en el contexto internacional, con énfasis en el ámbito universitario; para ello, se aplican técnicas cienciométricas a información obtenida de bases de datos de artículos científicos y técnicos. Así mismo, se examina el papel de las universidades, para lo cual se hace un análisis comparativo de algunos casos de reconocido impacto en el nivel mundial. Entre los resultados obtenidos está la identificación de áreas relevantes como la nanotecnología y la telefonía móvil, y la caracterización del manejo de las tecnologías emergentes en las universidades seleccionadas. Esto da la base para plantear algunas conclusiones y consideraciones, como la necesidad de que las universidades colombianas lideren el desarrollo tecnológico en el país promoviendo su impacto en el sector productivo, para lo cual requieren consolidar estructuras y políticas claras en el tema, así como una cultura en propiedad intelectual.

1 Facultad de Minas, Universidad Nacional de Colombia, Sede Medellín, cnjimenezh@unal.edu.co

2 Facultad de Ingeniería, Universidad Nacional de Colombia, Sede Bogotá, ofcastellanosd@unal.edu.co

3 Facultad de Minas, Universidad Nacional de Colombia, Sede Medellín, emvilla@unal.edu.co

Fecha de recepción: 12 de Enero de 2011

Fecha de aceptación: 26 de Julio de 2011 


\section{Palabras clave}

Tecnologías emergentes, ingeniería, universidades, transferencia tecnológica, desarrollo tecnológico.

\section{Abstract}

Some technologies considered emerging, have high uncertainly and particularities in their management respect to mature technologies. The aim of this paper is to analyze the management of emerging technologies, focused on the university context. For this purpose, scientometric techniques are applied to information from databases of technical papers. The role of universities is studied through a comparative analysis of some internationally recognized cases. As a result, relevant areas were identified, as nanotechnology and mobile communications, and management of emerging technologies in selected cases was characterized. These results are the base to formulate some conclusions and proposals for Colombian universities: They must lead technological development in the country through their impact on the industrial sectors, but these institutions need structures and clear policies, as well as an Intellectual Property culture.

\section{Keywords}

Emerging technologies, engineering, universities, technology transfer, technological development. 


\section{INTRODUCCIÓN}

Los procesos de generación, transferencia y aprovechamiento productivo de la tecnología en las economías emergentes de Latinoamérica, se han visto influenciados por la dinámica y creciente aceleración del factor conocimiento, lo que ha llevado a buscar el fortalecimiento de la tecnología como aspecto estratégico de las organizaciones y los países, tomando como base las capacidades de investigación y desarrollo existentes en la región, así como en el interés creciente en el trabajo en redes y en los esquemas de cooperación (Jiménez et al., 2007). Como parte de este proceso de fortalecimiento, es importante analizar si los logros de otros contextos pueden constituirse en opciones de desarrollo para América Latina.

En este sentido, es conocido el papel de las universidades en Estados Unidos y Europa, al facilitar que los resultados de los procesos de investigación y desarrollo obtenidos en el ámbito académico tengan aplicación en el sector productivo y al consolidar esquemas de integración como el Silicon valley en California o las biorregiones europeas, orientados a la creación de industrias basadas en conocimiento, para lo cual han generado estructuras y mecanismos apropiados para una gestión tecnológica de impacto real y concreto en el entorno en el cual se insertan (Rubiralta, 2004). Precisamente este papel preponderante de las universidades es uno de los pilares del nivel de desarrollo tecnológico y económico de las naciones industrializadas, y la ingeniería ha sido en gran parte el soporte fundamental para llevar a la práctica productiva los desarrollos que están en la frontera del conocimiento.

En el caso de las llamadas tecnologías emergentes, es decir, aquellas que potencialmente crearán nuevas industrias y que todavía no tienen un mercado consolidado, el rezago de países como Colombia es notorio. No obstante, el país puede aprender de dichas experiencias, teniendo en cuenta que en el tema de las tecnologías emergentes y su gestión, de acuerdo con Day et al. (2001), no son plenamente satisfactorios los enfoques tradicionales, por lo que es fundamental prepararse para hacer frente a los problemas derivados de la alta incertidumbre y el rápido cambio que caracterizan a las mencionadas tecnologías. 
Este artículo tiene como objetivo analizar la gestión de las tecnologías emergentes en el contexto internacional, enfatizando en el papel de las universidades en dicho proceso, y mostrando parte de los resultados de un proyecto de investigación sobre el tema. Para cumplir con este propósito, se desarrolló un estudio cienciométrico sobre artículos científicos y tecnológicos disponibles en bases de datos de revistas indexadas. Adicionalmente, se exploraron algunos casos de éxito de universidades de países desarrollados y referentes para Colombia. Se revisaron algunas características de la gestión tecnológica universitaria en Colombia, y por último, se formularon conclusiones a partir de los hallazgos y algunas consideraciones como aporte al papel de las universidades colombianas en la gestión tecnológica.

\section{PARTICULARIDAdeS DE LAS TECNOLOgíAS EMERgenteS $Y$ SU GESTIÓN}

Las tecnologías emergentes son aquellas que se encuentran en la fase inicial del ciclo de vida de la tecnología; nacen cuando surgen propuestas innovadoras de desarrollo de procesos, habilidades o aplicaciones diferentes que cambian las concepciones ya establecidas dentro del mercado y son capaces de modificar industrias ya constituidas y técnicas afianzadas (Day et al., 2001). Tales tecnologías pueden resultar de la intersección de ramas de investigación que antes estaban separadas (por ejemplo, los biocombustibles y la conectómica); pueden ser individuales como es el caso de la fibra óptica, o puede tratarse de grupos de tecnologías que convergen para crear un sistema tecnológico, como la Internet. Prácticamente en cualquier área se pueden encontrar ejemplos de tecnologías emergentes que transformaron a toda una industria: la robótica en la fabricación de los automóviles o el teléfono celular en las comunicaciones.

Dada su condición emergente, las tecnologías de este tipo se caracterizan por carecer de mercados constituidos y de datos históricos que faciliten la realización de análisis y pronósticos; así mismo, estos desarrollos son desconocidos, no probados, arriesgados y por tanto, difíciles de gestionar. Las organizaciones se enfrentan a 
la tarea de identificar las nuevas tecnologías, pero también al reto de desarrollarlas, gestionarlas y promover su uso (Bhattacherjee, 1998). La incorporación de estas tecnologías a los procesos productivos debe resultar en la generación de valor agregado e incremento de productividad, pero cuando se hace, estas tecnologías incipientes abren una serie de expectativas que tal vez no se logren. Day et al. (2001) afirman que una tecnología emergente puede ser arrolladora ya que puede acabar con los procesos, los productos, o la misma industria, citando como ejemplo a la Internet, capaz de derrumbar barreras para los competidores, crear nuevos modelos de negocio y nuevos mercados.

Por tanto, en el marco de la gestión tecnológica, las tecnologías emergentes tienen un espacio particular debido a sus características distintivas asociadas al riesgo, la incertidumbre y la falta de datos históricos, como ya se indicó, y dado su alto potencial, una eficaz gestión puede llegar a hacer la diferencia entre el logro de impactos radicales como los de la Internet o la nanotecnología, o un esfuerzo que no trascienda el ámbito científico y académico.

\section{TENDENCIAS EN LA GESTIÓN DE TECNOLOGÍAS EMERGENTES}

Con el fin de determinar tendencias en la gestión de tecnologías emergentes, se realizó un análisis de la literatura sobre el tema consultada a través del Sistema Nacional de Bibliotecas de la Universidad Nacional de Colombia. Esta revisión de literatura se realizó mediante un análisis cienciométrico a partir de la información de tres bases de datos de artículos científicos y técnicos (ScienceDirect, ISI Web of knowledge y Academic Search Complete). Se empleó la ecuación de búsqueda manag* AND "emerging technolog*", que reúne los dos descriptores principales del tema de interés y sus posibles variantes. Luego de una depuración para evitar duplicidades e información no pertinente, se trabajó con 98 referencias, que fueron descargadas al programa Reference Manager y los indicadores cienciométricos se generaron con Excel (tablas) y RefViz (mapa de proximidades).

La tendencia de publicación de artículos en el tema de la gestión de tecnologías emergentes ha sido creciente en los últimos 
años, siendo 2008 el año en el que se encontró la mayor cantidad de documentos (36 registros). La Tabla 1 presenta la frecuencia de aparición de algunas de las palabras clave citadas en tales artículos durante el periodo analizado. La ventana de observación o periodo de búsqueda se dividió en dos intervalos (1999 a 2004 y 2005 a 2009) para identificar las áreas crecientes y nacientes. Las áreas crecientes, que se muestran en la primera columna de la Tabla 1, se refieren a aquellas palabras clave más citadas en los documentos revisados, y cuya frecuencia de aparición es mayor en el segundo intervalo de tiempo que en el primero. Teniendo en cuenta que "technological innovations" es la palabra clave más frecuentemente citada en los dos intervalos, se puede afirmar que las tecnologías emergentes se relacionan principalmente con innovaciones de tipo tecnológico, lo que indica que muchas de estas tecnologías, al ser gestionadas, tienen éxito en el mercado, según la definición de innovación tecnológica establecida en el Manual de Oslo (OCDE, 2005).

Tabla 1. Áreas relevantes en gestión de tecnologías emergentes

\begin{tabular}{|c|c|c|}
\hline \multirow{2}{*}{ Áreas crecientes } & \multicolumn{2}{|c|}{ Artículos en el periodo } \\
\hline & 1999-2004 & 2005-2009 \\
\hline Technological innovations & 19 & 53 \\
\hline Nanotechnology & 1 & 13 \\
\hline Health care & 2 & 9 \\
\hline Internet Technologies & 4 & 6 \\
\hline Biology & & 9 \\
\hline Risk Management & 1 & 6 \\
\hline \multirow{2}{*}{ Áreas nacientes } & \multicolumn{2}{|c|}{ Artículos en el periodo } \\
\hline & $1999-2004$ & 2005-2009 \\
\hline Forecast of technology breakthroughs & 1 & 5 \\
\hline Decision support Systems & & 5 \\
\hline Electronics & & 5 \\
\hline Technology evaluation & 1 & 4 \\
\hline Mobile communication Systems & 2 & 3 \\
\hline Patent analysis & 1 & 3 \\
\hline
\end{tabular}


Esta área de innovación tecnológica tuvo un crecimiento notorio en el periodo analizado, lo cual señala el énfasis que se ha dado a la necesidad de que los desarrollos tecnológicos, si bien tienen alta incertidumbre en su fase inicial, apunten a convertirse en innovaciones. Precisamente el tema de gestión del riesgo asociado a las tecnologías emergentes debido a su incertidumbre, también es un área creciente según lo presentado en la Tabla 1. Además puede apreciarse que una de las tecnologías emergentes más estudiada en cuanto a su gestión es la nanotecnología, así como las tecnologías asociadas a la vida en temas como el cuidado de la salud y la biología, y la Internet, que continúa revolucionando la forma de hacer las cosas.

La Tabla 1 también presenta las áreas nacientes, aquellas en las cuales se está comenzando a investigar, asociadas con la gestión de tecnologías emergentes. Estas áreas se identificaron mediante las palabras clave poco citadas en las que se evidencia una marcada tendencia creciente entre un intervalo de tiempo y otro. En dichas áreas se encuentra la electrónica, los sistemas de comunicación móvil y los sistemas de soporte a las decisiones, cuya dinámica actual puede estar relacionada con la introducción de tecnologías emergentes. Por otra parte, se observa el naciente interés en actividades fundamentales para la gestión de este tipo de tecnologías, como el pronóstico de avances tecnológicos radicales, la evaluación de tecnologías y el análisis de patentes.

La Fig. 1 muestra el mapa de proximidades o galaxia generada mediante el programa RefViz, seleccionado porque facilita el análisis de grandes cantidades de información, ya que agrupa los documentos a partir de la relación existente entre sus contenidos, con base en la estructura semántica y sintáctica en los títulos y los resúmenes, y caracteriza cada agrupamiento por medio de un conjunto de términos descriptivos (www.refviz.com). Dicha galaxia presenta una forma ovoide y alargada, lo cual indica la contribución múltiple de varios temas, que se evidencia por la cantidad de clusters generados: en este mapa se identifican nueve clusters que a su vez pueden analizarse en tres áreas según la afinidad entre los 98 artículos encontrados. El área A involucra referencias asociadas con el análisis de las tendencias futuras de las tecnologías emergentes, el desarrollo de mercados para estas tecnologías y el 
estudio de los factores que afectan tales mercados. El área B reúne artículos relacionados con métodos, procesos y modelos de gestión de tecnologías emergentes. Finalmente, el área $\mathrm{C}$ asocia referencias sobre diversas aplicaciones de las tecnologías emergentes.

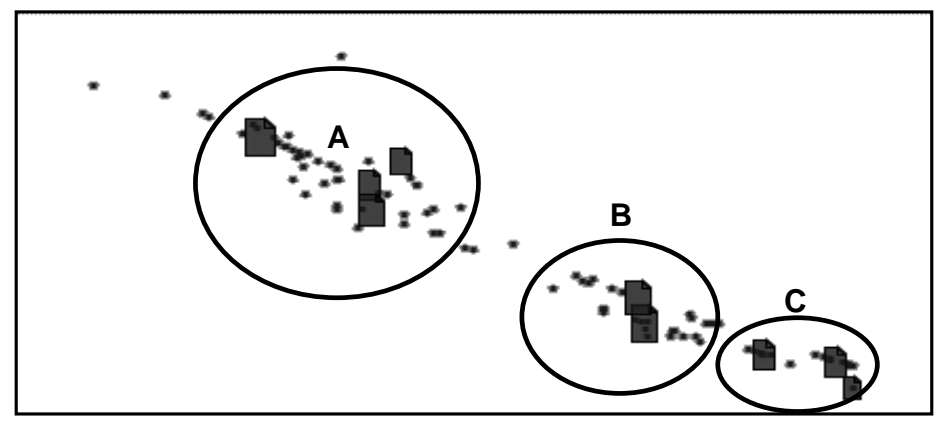

Fig. 1. Mapa de proximidades en gestión de tecnologías emergentes Análisis a partir de la información de las bases de datos ScienceDirect, ISI Web of knowledge y Academic Search Complete, Periodo 1999-2009, Fecha de búsqueda: 22/05/09.

\section{LA GESTIÓN DE TECNOLOGÍAS EMERGENTES EN EL ÁMBITO UNIVERSITARIO}

Debido a que las tecnologías emergentes pueden originarse, en muchos casos, en el marco de proyectos académicos de investigación tecnológica, son los grupos y centros de investigación de instituciones universitarias los que, por lo general, lideran el proceso desde el surgimiento de la idea hasta lograr una transferencia exitosa del desarrollo tecnológico al mercado. A partir de este hecho, se hizo un segundo ejercicio revisión de literatura, orientado a conocer el papel de los grupos de investigación universitarios en la gestión de tecnologías emergentes. Al igual que en el caso anterior, esta revisión se realizó mediante un análisis cienciométrico utilizando la ecuación de búsqueda Manag* AND "Emerg* Technologies" AND R\&D AND university groups, la cual limitó la primera búsqueda a aquellos artículos relacionados con grupos universitarios de investigación y desarrollo. Las bases empleadas fueron ScienceDirect, ISI Web of knowledge y Academic Search Complete, 
y se encontraron 13 registros en total, divulgados entre 2000 y 2006.

En la Tabla 2 se presenta la dinámica o frecuencia de aparición en el periodo estudiado de las palabras clave más citadas en los artículos encontrados. En general, estas palabras se asocian con los procesos de gestión de las tecnologías que generan las universidades, tales como Research Management, Management of Technology Transfer y university-industry relationship. El área de Determinants on research progress es mencionada en artículos que enfatizan en el papel de la universidad como generadora de conocimiento y promotora del desarrollo tecnológico. También se destaca el área de innovation policy, la cual tiene una tendencia creciente en los artículos analizados, evidenciando que los grupos de investigación y las universidades que los respaldan, progresivamente han asumido un papel más relevante al orientar su actividad investigativa a generar impactos en la sociedad, es decir, se han enfocado en la creación de innovaciones.

Tabla 2. Dinámica de áreas relevantes en gestión de tecnologías emergentes en universidades

\begin{tabular}{|c|c|c|c|c|c|c|c|}
\hline \multirow{2}{*}{ Áreas relevantes } & \multicolumn{7}{|c|}{ Cantidad de artículos } \\
\hline & 2000 & 2001 & 2002 & 2003 & 2004 & 2005 & 2006 \\
\hline Research management & 2 & 7 & 7 & & 3 & 1 & 4 \\
\hline Management of technology transfer & 1 & & 12 & 3 & & & 4 \\
\hline Determinants on research progress & & 7 & 7 & & & 1 & \\
\hline Innovation policy & 2 & & 2 & 3 & & 2 & 4 \\
\hline University-industry relationships & 3 & & 1 & 3 & 3 & 2 & \\
\hline
\end{tabular}

Análisis a partir de la información de las bases de datos ScienceDirect, ISI Web of knowledge y Academic Search Complete, Periodo 1999-2009, Fecha de búsqueda: 18/09/09.

\subsection{Análisis Comparativo de Universidades Líderes}

Como complemento de la revisión de literatura presentada, se realizó un análisis comparativo de varias universidades reconocidas internacionalmente por su actividad científica y tecnológica. Para su elección se tomaron en cuenta tres aspectos: 1) inclusión en los primeros 300 lugares del Academic Ranking of World Universities 2009, que se basa en indicadores como premios Nobel y 
reconocimientos equivalentes, artículos publicados en revistas de Nature y Science, artículos indexados en el Science Citation Index y el Social Sciences Citation Index, así como el desempeño per cápita respecto al tamaño de cada institución (ARWU, 2009). 2) Ubicación de estas universidades en países industrializados o en economías emergentes latinoamericanas que son de referencia para Colombia. 3) Disponibilidad de información sobre tales instituciones, principalmente en sus páginas web. De esta forma se trabajó con las siguientes universidades: la Universidad de Pensilvania (UPENN), el Instituto de Tecnología de Massachusetts (MIT), ambas instituciones en los Estados Unidos, la Universidad de la Columbia Británica de Canadá (UBC), la Universidad de Cambridge, Reino Unido (UCAM), la Universidad Estatal de Campinas, Brasil (UNICAMP), la Universidad Politécnica de Valencia, España (UPV) y la Universidad Nacional Autónoma de México (UNAM). El análisis comparativo se realizó tomando en cuenta varios aspectos respecto a la generación y gestión de tecnología en las universidades, haciendo énfasis en las tecnologías emergentes. Las Tablas 3 y 4 resumen los hallazgos.

En la Tabla 3 se presentan los aspectos generales que caracterizan las universidades seleccionadas, así como su actividad investigativa y de generación de tecnologías. En general, puede observarse que las elevadas capacidades en investigación y obtención de resultados se basan en la disponibilidad de recursos financieros y físicos (obviamente además del talento humano), las amplias y variadas relaciones de cooperación e intercambio, la interacción con un el entorno y la existencia de políticas internas y gubernamentales de apoyo a las actividades de ciencia y tecnología.

LaTabla 4 resume los aspectos específicos en la gestión de las tecnologías generadas por las universidades, con énfasis en las tecnologías emergentes. Es muy relevante el hecho de que en cada universidad exista una oficina exclusivamente dedicada a dicha gestión, que en algunos casos se constituye como un ente aparte de la estructura universitaria, pero que siempre dispone de las herramientas para apoyar a los investigadores en el proceso de convertir las invenciones en innovaciones.

Dentro de las herramientas con las que cuentan estas oficinas, se encuentran lineamientos procedimientos definidos en temas 
como propiedad intelectual y negociación. Incluso se pudo identificar mecanismos concretos orientados a las tecnologías emergentes, evidenciando que las universidades reconocen sus particularidades y por tanto, su trato diferencial. También puede resaltarse la existencia de grupos de investigación y programas puntuales en torno al tema de tecnologías emergentes, de manera que las universidades preparan a los futuros profesionales y asesoran a los empresarios frente al reto de gestionar estas tecnologías.

\subsection{El Caso Colombiano}

De acuerdo con López et al. (2006), las patentes de invención concedidas a universidades colombianas representan el $0,94 \%$ de las otorgadas a residentes y $0,007 \%$ del total concedido. Si bien estas cifras muestran un panorama poco alentador, en años recientes en el país se han generado importantes dinámicas en torno al desarrollo tecnológico liderado por las universidades, que han conllevado algunos resultados en la interacción con la industria. Con base en diversos documentos que han estudiado la forma en que las universidades colombianas gestionan sus avances tecnológicos con el fin de contribuir a la solución de problemas de la sociedad, Jiménez \& Castellanos (2008) encontraron que la región antioqueña es líder en estos procesos y los resultados e impactos son tangibles.

En dicho estudio se evidenció que las principales falencias en las universidades colombianas están relacionadas con la ausencia de políticas específicas en el tema y formuladas de manera explícita, y el hecho de que las unidades que responden por la gestión tecnológica no se dedican exclusivamente a ello, ya que además deben atender otros compromisos (como es el caso de las Direcciones de Investigación o Extensión), pero se resaltan casos de éxito como el Programa de Gestión Tecnológica de la Universidad de Antioquia. 
Tabla 3. Resumen comparativo de las universidades analizadas: Generalidades

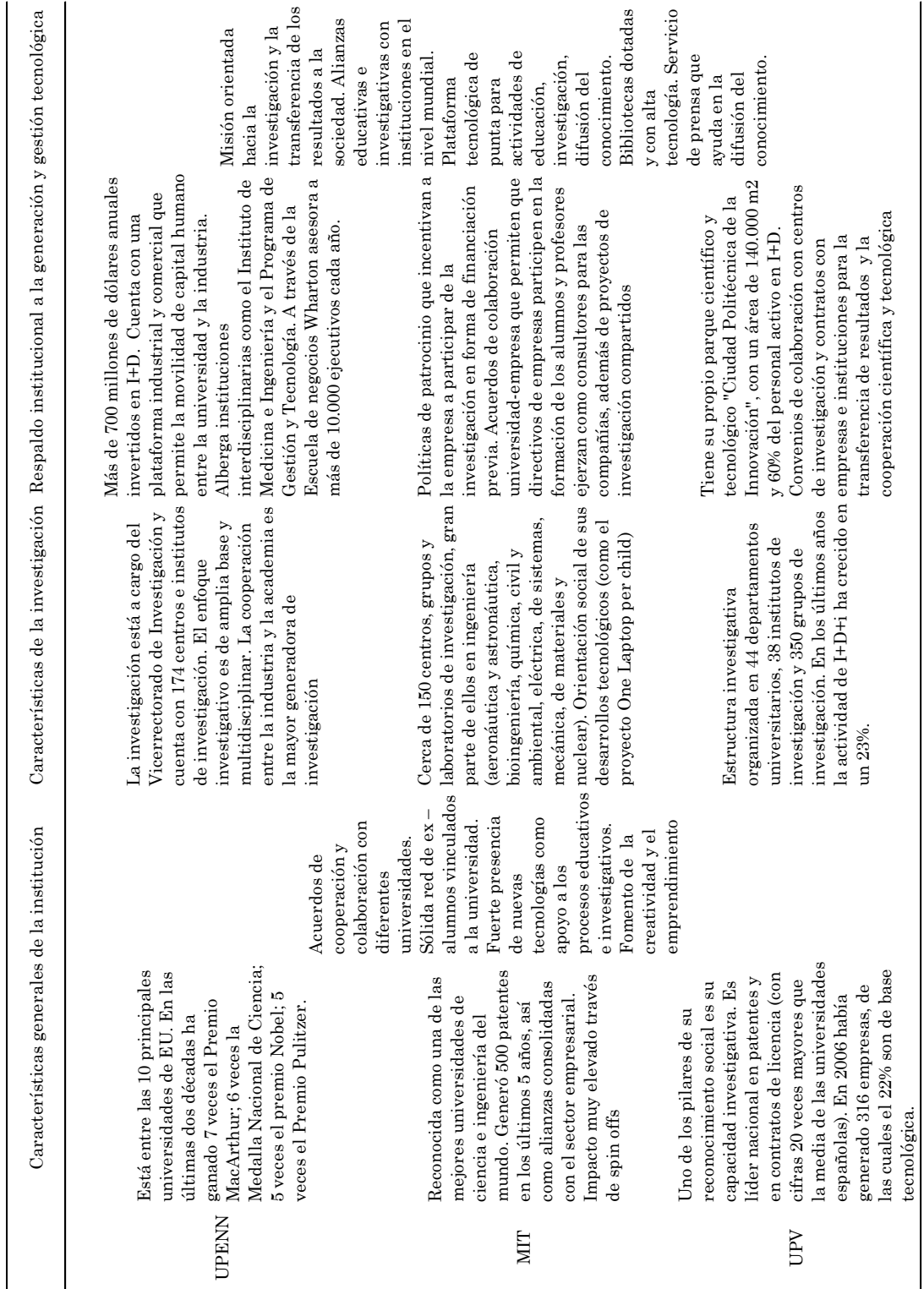

Recopilación con base en la información disponible en las páginas web de las universidades seleccionadas. 


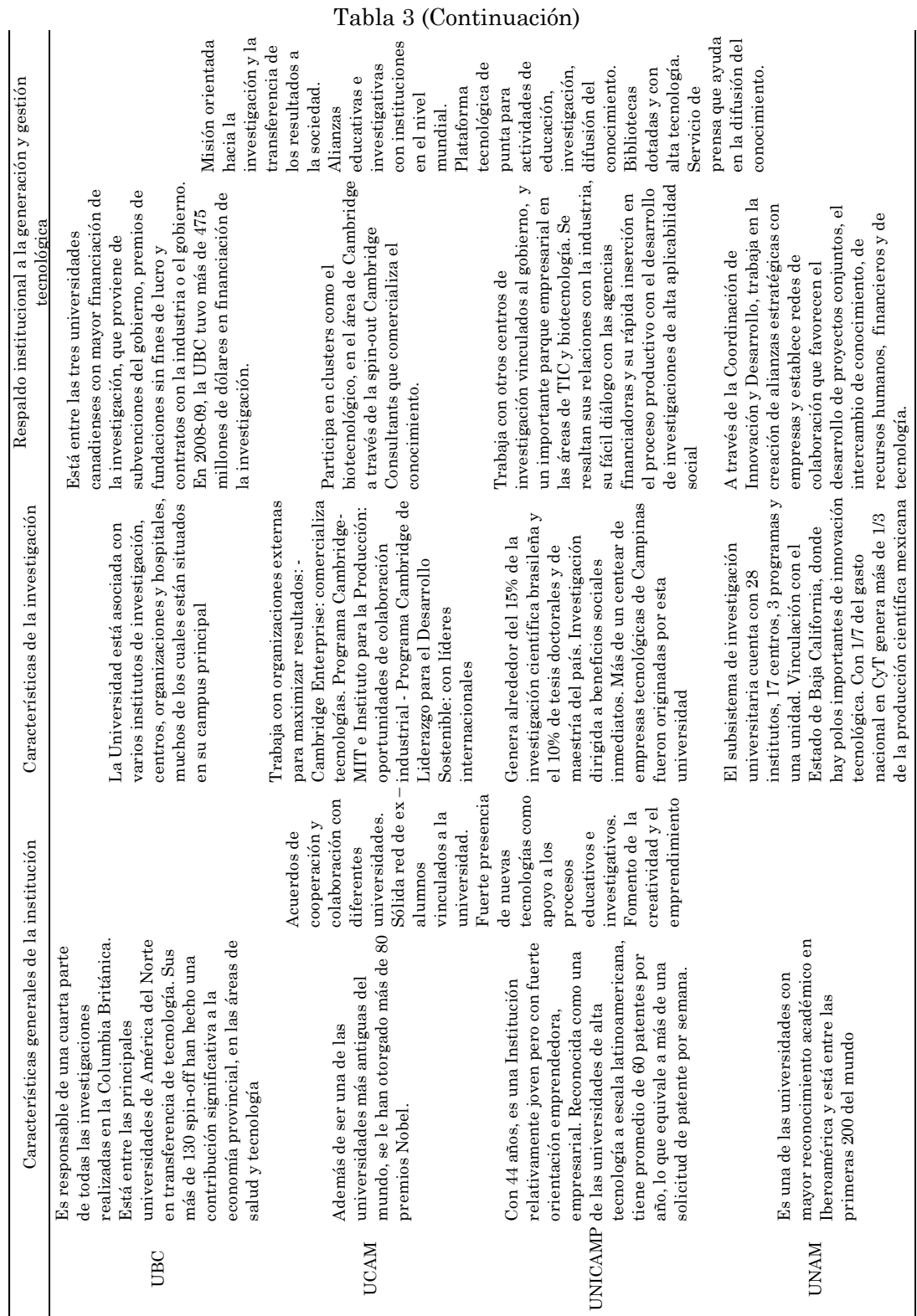

Recopilación con base en la información disponible en las páginas web de las universidades seleccionadas. 
Tabla 4. Resumen comparativo de las universidades: Gestión de tecnología

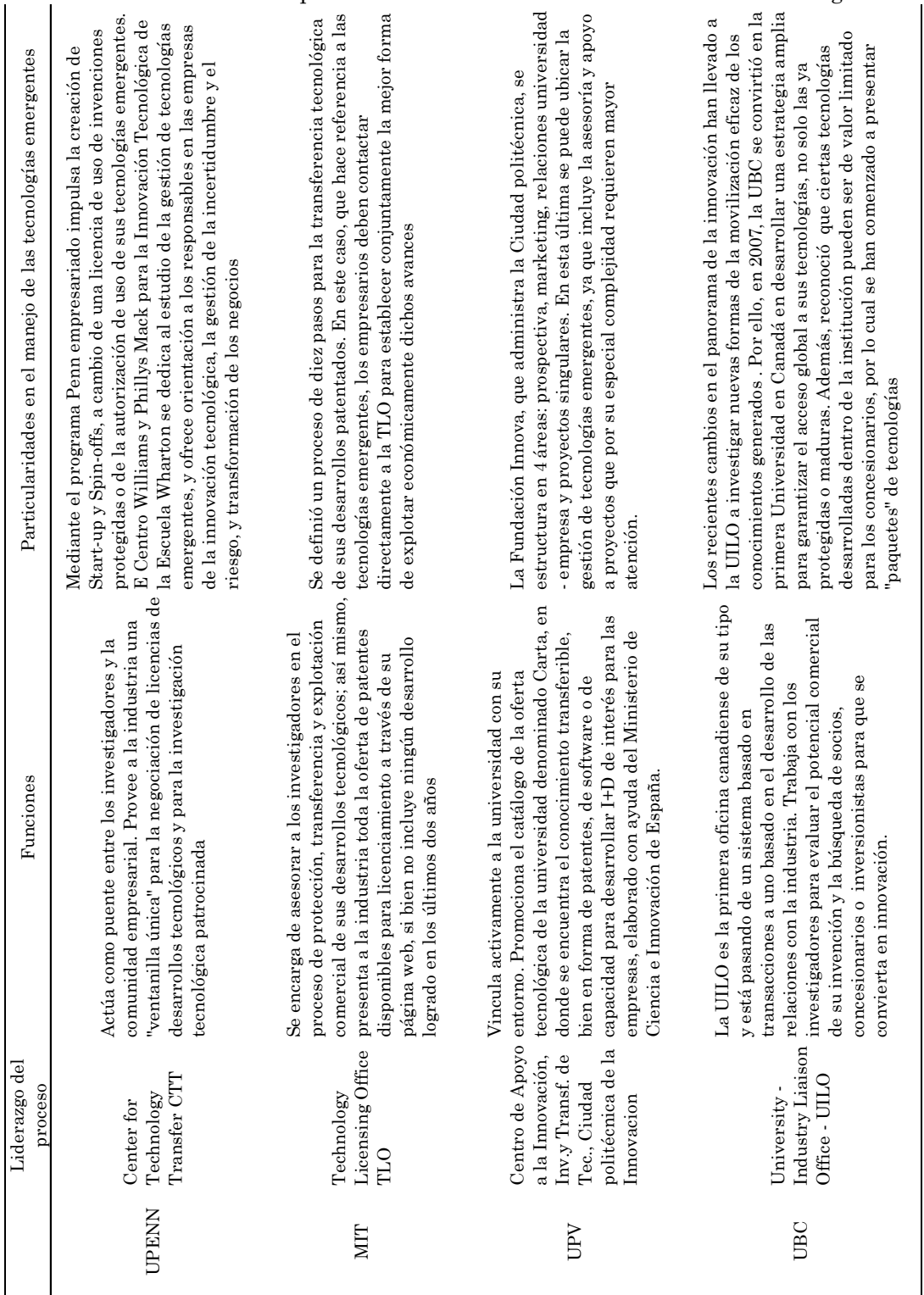

Recopilación con base en la información disponible en las páginas web de las universidades seleccionadas. 


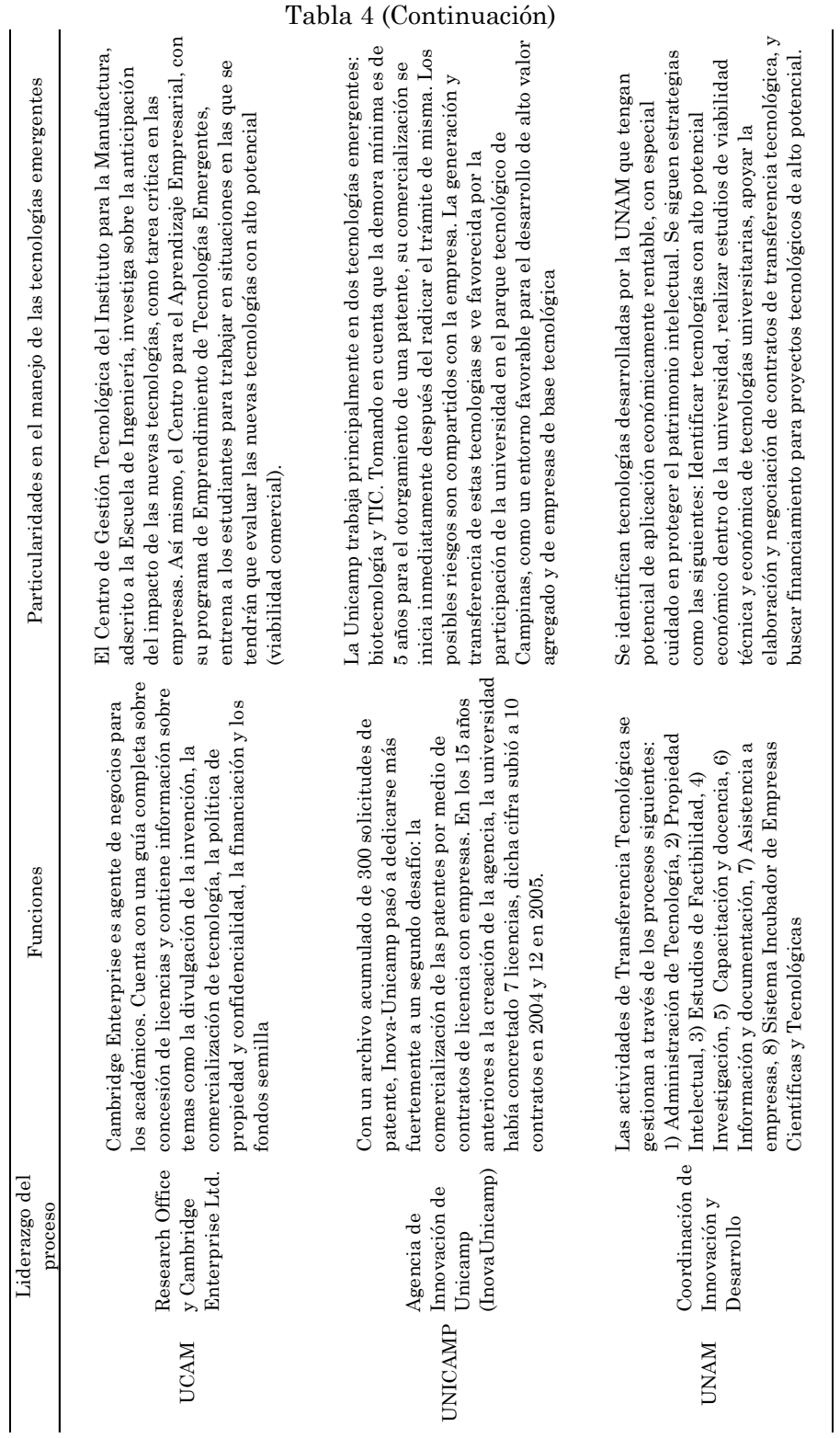

Recopilación con base en la información disponible en las páginas web de las universidades seleccionadas. 
También se destacan iniciativas interuniversitarias como el Comité Universidad - Empresa - Estado y la fundación Tecnnova, que han aportado espacios y actividades de comunicación entre la academia, los empresarios y el gobierno, siendo un primer paso en la identificación de demandas y ofertas, la formulación conjunta de proyectos y la atención de necesidades en entornos regionales (Antioquia, Bogotá región). Al respecto, el estudio citado concluye que las universidades con estructuras y programas definidos en gestión tecnológica logran una mejor articulación en este tipo de iniciativas interuniversitarias, mostrando que el paso inicial debe ser la consolidación de la gestión tecnológica al interior de cada universidad para luego si avanzar hacia otros niveles (ciudad, región y país). Si bien el estudio referenciado no se orientó a las tecnologías emergentes, este tema no aparece planteado explícitamente en ninguna de las universidades colombianas revisadas.

\section{CONCLUSIONES Y CONSIDERACIONES FINALES}

Las tecnologías emergentes han ganado progresivamente un espacio dentro de la investigación y la transferencia tecnológica, reconociéndose sus características distintivas asociadas a su alto grado de incertidumbre, a la poca información relativa a ellas, y su innegable impacto en el desarrollo industrial, de manera que la investigación en la gestión de este tipo de tecnologías se ha enfocado en aplicaciones relevantes para la industria como la nanotecnología y las comunicaciones, y también en aspectos relacionados con el bienestar como las aplicaciones biológicas en salud.

Como se vio al identificar las áreas crecientes en el análisis cienciométrico, existe una relación directa entre las tecnologías emergentes y la innovación tecnológica. En este sentido, la generación y gestión de tecnologías debe apuntar al logro de impactos en el mejoramiento de procesos o productos. Por tanto, la investigación tecnológica llevada a cabo principalmente por los grupos de ingeniería debe estar apoyada institucionalmente por las universidades, de manera que se garantice su aplicación y explotación industrial, para lo cual la vinculación universidad - empresa resulta fundamental. La universidad de hoy está en un ambiente 
donde su papel puede ser fácilmente medido según su impacto en la sociedad, y es justamente este sentido de servicio, contemplado en su misión, el que ha hecho que los mecanismos de comunicación e interacción con el medio sean cada vez más proactivos. El ejercicio cienciométrico que reveló áreas relevantes en la gestión de tecnologías emergentes en las universidades, como la gestión de la investigación y la evaluación de tecnologías, confirma que este asunto es un tema de interés para los investigadores actualmente y que ocurre en diferentes instituciones educativas del contexto internacional.

El apoyo institucional a los grupos de investigación tecnológica también se observó en la revisión de los casos universitarios, en donde se halló una tendencia hacia la centralización de la gestión de tecnologías en las universidades, en una unidad u oficina que articula y dinamiza procesos, coordinando la implementación de las políticas en el tema, fortaleciendo y apoyando el trabajo que se hace desde cada facultad, instituto y grupo de investigación. De igual manera se resalta la definición de procedimientos relacionados con la transferencia de las tecnologías universitarias, si bien el aspecto más destacado es la existencia de lineamientos y normativas como el reglamento de propiedad intelectual, que es un aspecto abordado de manera explícita y soporta el desarrollo práctico de la vinculación universidad - empresa.

Sin desconocer los grandes aportes al desarrollo industrial del país que han hecho las universidades colombianas a través de sus grupos de investigación, es necesario considerar el hecho de que indicadores como la generación y licenciamiento de patentes o la creación de spin offs que presentan universidades extranjeras como las analizadas, aún no se han logrado en nuestro contexto. Por ello, puede afirmarse que las características identificadas en este artículo respecto a la generación y gestión de tecnologías emergentes en las universidades, se constituyen en retos para muchas instituciones colombianas que reconocen su papel como generadoras de conocimiento tecnológico y su compromiso con el progreso del país a través de su actividad investigativa, pero que aún no han logrado consolidar mecanismos que faciliten la transferencia de sus avances a la industria. 
Finalmente, en el marco del desarrollo industrial del país, es importante que la universidad colombiana genere soluciones tecnológicas en áreas prioritarias para Colombia y con un enfoque social, tomando en cuenta las tendencias internacionales, en donde las instituciones universitarias son las llamadas a ser líderes en campos como la nanotecnología, las telecomunicaciones, la electrónica, los materiales y la biotecnología, con la participación y financiación decidida del sector empresarial. Estas soluciones deberán estudiarse también desde el punto de vista del riesgo y del mercado, como temáticas relevantes según la cienciometría presentada en este artículo. Pero además, las universidades deben buscar que estas tecnologías impacten realmente como innovaciones, y fortalecer la cultura de propiedad intelectual al interior de los grupos de investigación, para lo cual requerirán desarrollar un sistema universitario que acompañe e incentive a los docentes y estudiantes a formular y ejecutar proyectos de investigación orientados a las empresas.

\section{AGRADECIMIENTOS}

Este artículo es resultado de un proyecto de investigación financiado con recursos de la Vicerrectoría de Investigación y de la Dirección de Investigaciones de la Sede Medellín (DIME), de la Universidad Nacional de Colombia.

\section{REFERENCIAS}

ARWU, (2009); Academic Ranking of World Universities 2009, extraído el 2 de abril de 2009, disponible en: http://www.arwu.org/

Bhattacherjee, A., (1998); Management of Emerging Technologies: Experiences and lessons learned at US West. Information and Management, 33(5), 263-272.

Day, G., Schoemaker, P., Gunther, R., (2001); Gerencia de Tecnologías Emergentes, Editorial Vergara Business, Buenos Aires, Argentina. 
Jiménez, C., Castellanos, O., (2008); Desafíos en gestión tecnológica para las universidades como generadoras de conocimiento, I Congreso internacional de Gestión Tecnológica e Innovación, Bogotá.

Jiménez, C., Castellanos, O., Fonseca, S., (2007); Gestión tecnológica: de un enfoque tradicional a la gestión del conocimiento. Consideraciones y retos para Latinoamérica, XII Seminario Latino Iberoamericano de Gestión Tecnológica ALTEC, Argentina.

López, M., Schmal, R., Cabrales, F. (2006); Comportamiento de las patentes universitarias: Colombia, Chile y España, extraído el 19 de junio de 2010, disponible en:

www.faceaucentral.cl/pps/presentacion_colombia.ppt

Massachusetts Institute of Technology, (2009); página web del MIT, extraído el 12 de abril de 2009, disponible en www.web.mit.edu

OCDE, (2005); Manual de Oslo: Guía para la recogida e interpretación de datos sobre innovación (versión traducida), Grupo TRAGSA, España.

Rubiralta, M., (2004); Transferencia a las empresas de la investigación universitaria. Descripción de modelos europeos, Fundación Cotec para la innovación tecnológica, España.

Universidad Nacional Autónoma de México, (2009); página web de la UNAM, extraído el 20 de junio de 2009, disponible en: www.unam.mx

Universidad Politécnica de Valencia, (2008); página web de la Universidad Politécnica de Valencia, extraído el 15 de mayo de 2009, disponible en: http://www.upv.es/carta/

Universidade Estadual de Campinas, (2009); página web de la Universidade Estadual de Campinas, extraído el 30 de junio de 2009, disponible en: www.unicamp.br

University of British Columbia, (2008); página web de la Universidad de la Columbia Británica, extraído el 22 de abril de 2009, disponible en: www.ubc.ca

University of Cambridge, (2009); página web de la Universidad de Cambridge, extraído el 30 de abril de 2009, disponible en: www.cam.ac.uk

University of Pennsylvania, (2008); página web de la Universidad de Pensilvania, extraído el 18 de mayo de 2009, disponible en: www.upenn.edu 
